\title{
La liaison langage - lecture dans quelques manuels en usage en Afrique
}

\section{Michel Verdelhan}

\section{(2) OpenEdition \\ 12 Journals}

Édition électronique

URL : http://journals.openedition.org/trema/2184

DOI : 10.4000/trema.2184

ISSN : 2107-0997

\section{Éditeur}

Faculté d'Éducation de l'université de Montpellier

\section{Édition imprimée}

Date de publication : 1 juin 1995

Pagination : 47-54

ISSN : 1167-315X

\section{Référence électronique}

Michel Verdelhan, «La liaison langage - lecture dans quelques manuels en usage en Afrique », Tréma

[En ligne], 7 | 1995, mis en ligne le 23 septembre 2013, consulté le 19 avril 2019. URL : http:// journals.openedition.org/trema/2184; DOI : 10.4000/trema.2184

Ce document a été généré automatiquement le 19 avril 2019

Trema 


\title{
La liaison langage - lecture dans quelques manuels en usage en Afrique
}

\author{
Michel Verdelhan
}

1 Le présent travail a pour objet l'examen de la liaison entre l'enseignement du langage et celui de la lecture, liaison revendiquée par la plupart des manuels de français destinés aux premières classes - cours d'initiation et cours préparatoire - de l'école primaire dans les pays de l'Afrique francophone.

2 C'est sans doute le premier problème pédagogique que doit résoudre l'enseignement du français langue seconde. Titres ou sous-titres, préfaces ou avant-propos attestent de cette volonté de lier les deux enseignements. Et de fait, sans attendre que les recherches théoriques des psychologues aient dissipé totalement le mystère des relations entre le processus d'apprentissage du langage et celui de la lecture, les manuels récents en usage en Afrique établissent selon diverses modalités une liaison entre les deux domaines. L'étude portera sur la douzaine de manuels suivants :

- Piga et Bika, CP1 et CP2, EDICEF-NEA., Gabon, 1984-1985.

- A toi de parler! Hatier-EDICEF, (Madagascar), 1985.

- Mon livre unique de français, Langage-lecture, Alwine Ekotto Ebolo, CEPER. EDICEF, Cameroun, 1988.

- Pour apprendre le français, Première année et deuxième année, B.E.R. EDICEF, Burundi, 1989.

- Langage, INEADE, Sénégal, 1990.

- Sidi et Rama, INEADE, Sénégal, 1990.

- Paroles et images, Larousse, Zaïre, 1991.

- Le Français par les images, C.I. EDICEF, République Centrafricaine, 1991.

- Le Flamboyant, CP1, Hatier, 1991.

- La Pirogue, CP1, EDICEF, 1993.

- Le Cabri et le Chacal, CI et CP, EDICEF, Djibouti, 1993 et 1994.

- Langage en fête, CP1, EDICEF, 1993.

Ce corpus ne prétend pas à l'exhaustivité des manuels en usage. Il se limite aux ouvrages parus ces dix dernières années. Mais en Afrique certains manuels ont une très longue vie. 
Si le célèbre Mamadou et Bineta a été encore récemment réédité, c'est qu'il est toujours utilisé ici ou là, près de cinquante ans après sa parution. De plus, un manuel peut en cacher un autre: Pour apprendre le français est une adaptation au Burundi de l'ouvrage camerounais Mon livre unique de français. La Pirogue CP1 reprend pour le langage les supports iconiques de ce même ouvrage camerounais, à quelques détails près. Ce corpus réunit aussi des ouvrages spécifiques pour un pays donné, comme Le Cabri et le Chacal, et des livres à vocation panafricaine comme Le Flamboyant, La Pirogue, Langage en fête.

Enfin il ne reflète pas forcément la pratique de ces enseignements liés du langage et de la lecture. En Afrique encore plus qu'ailleurs le fossé peut être grand qui sépare la réalité vécue de la classe et ce que le manuel pouvait en laisser prévoir.

Nous ne cherchons donc pas à rendre compte de l'enseignement du français en Afrique francophone dans les deux premières années de scolarité mais plus modestement nous allons cerner les types de solutions envisagés par les méthodologues pour lier langage et lecture. Ces solutions intéressent tous ceux qui en France sont amenés, pour des raisons diverses, à conduire, la même année, dans leur classe, ce double enseignement du langage et de la lecture à des élèves d'origine étrangère. Bien sûr, il ne s'agit pas de proposer à nouveau le transfert d'une méthodologie du français langue étrangère dont le français langue seconde n'est sans doute qu'un cas particulier. Il n'en demeure pas moins que les maîtres qui ont à enseigner, à parler et à lire à des élèves étrangers, ont bien à résoudre le même genre de problèmes que les enseignants des C.I. et C.P. africains.

La méthode suivie dans cet inventaire des types de liaison langage-lecture consiste à confronter les déclarations d'intention des auteurs avec l'organisation concrète de cette liaison dans le manuel. Cette liaison s'instaure selon diverses modalités, elle va de la juxtaposition à de profondes imbrications en passant par des articulations plus ou moins superficielles.

\section{Les juxtapositions}

7 Rares sont les ouvrages qui avouent l'absence de lien entre deux enseignements du parler et du lire simplement juxtaposés. Le manuel Le français par les images a de ce point de vue le mérite de la franchise. Parmi "quelques conseils pratiques» du livre du maittre, on trouve le constat lucide d'« un écart entre le contenu des leçons de langage et celui des phrases clés et des textes de lecture ». Le manuel ne cherche nullement à combler cet écart » reconnu : les auteurs comptent sur « un enseignement interdisciplinaire » et sur « les situations de la vie courante » pour prolonger l'enseignement du langage et rendre possible la compréhension des textes de lecture.

8 Généralement les avant-propos affirment avec d'autant plus de force la liaison langagelecture que le livre de l'élève ne parvient pas à l'instaurer. Ainsi par exemple, le manuel pour le CP2 de la collection Piga et Bika annonce « dans la deuxième partie ... des textes en rapport avec les leçons de langage ». Mais les élèves auront bien du mal à percevoir ces rapports. On retrouve seulement assez souvent les mêmes personnages dans les deux leçons. Mais la phrase clé est en général la légende d'une image sans rapport avec le dialogue de la leçon de langage.

Exemple:

Dialogue :- Donne-moi un livre.- Le voici, prends-le.Phrase clé:Le docteur soigne Bika. 

d'Etude et d'Action pour le Développement de l'Education, Dakar), Langage et Sidi et Rama. Quand le dialogue par exemple concerne l'école, la phrase à lire aussi. Mais les auteurs comptent sur la possibilité «d'élargir au maximum ... l'exploitation des thèmes langagiers » pour "régler le problème de la liaison langage-lecture sans verser dans le psittacisme qui résulterait d'une trop forte ressemblance entre les deux manuels ». Toutefois, avec cette série d'ouvrages, les maitres risquent de ne pas savoir comment " élargir l'exploitation des thèmes langagiers », le guide pédagogique ne fournissant pas d'indication pour cela. On accordera aux auteurs une claire conscience du problème : aux praticiens de trouver la solution!

\section{Les répétitions}

Tous les ouvrages n'évitent pas le psittacisme dans lequel les didacticiens de Dakar n'ont pas voulu verser. Ainsi par exemple, Paroles et Images, après avoir assuré la lecture des consignes, propose tout simplement, comme dans les plus anciennes méthodes de Français Langue Etrangère, de lire des extraits du dialogue répété pendant la leçon de langage, sans aucun souci de transposition dans une situation d'écrit.

Voici un échantillon de ces phrases d'oral retenues comme support de lecture :

« C'est mon père. Oui, c'est mon père.

C'est ma mère. Regarde, c'est ma mère. »

La reproduction littérale de l'oral atteint un caractère parfaitement artificiel avec cette série qui représente le programme de lecture de quatre leçons :

"C'est le matin, il est 7 heures.

C'est le matin, il est 11 heures.

C'est l'après midi, il est 15 heures.

C'est le soir, il est 20 heures. »

14 Si l'horloge parlante émettait des messages écrits, avec une telle méthode, les élèves sauraient les lire !Les seuls écrits véritables apparaissent dans la quatrième et dernière unité avec la légende des images.

\section{Une liaison texte-image : la légende-charnière}

15 La solution qui consiste à utiliser la légende de l'image - à laquelle aboutissent les échanges oraux - pour assurer la liaison avec la leçon de lecture est choisie par plusieurs manuels récents. On la retrouve dans La Pirogue CP1 et dans Le Flamboyant CP1. Mais cette solution est en réalité très ancienne : c'était celle de Mamadou et Bineta (ISTRA, 1950) qui précisait pour le maître : «montrer et faire dire ... » par exemple « un canari, un épi » (leçon 1). Pour parler français, Première année (CLAD. EDICEF, 1967) avait recours à peu près à ce même procédé de la légende comme introduction de la phrase à lire.

16 Evidemment l'image est là pour assurer la compréhension de la phrase. En conséquence la phrase est à l'égard de l'image parfaitement redondante. On a vigoureusement dénoncé en son temps le fait que pour accompagner la phrase: «Doudou est sous le filao » (Pour parler français, p. 5), l'image montre Doudou et le filao. On retrouve cependant le même 
procédé dans les manuels plus récents. L'énoncé : «Des gazelles se reposent sous un arbre » s'insère sous un dessin représentant des gazelles allongées sous un arbre! (Piga et Bika, CP1, p. 48) (fig. 1) Le flou énonciatif de la phrase (Qui parle? A qui ?) va de pair avec son inutilité communicative. Dire ce que l'image a déjà dit dans sa transparence pédagogique est déjà vain, le lire ensuite c'est bien tomber dans ce psittacisme dénoncé par l'INEADE.

On le voit, la liaison langage-lecture que les manuels postulent pour les premières années d'enseignement du français n'est pas facile à réaliser quand on ne veut pas tout simplement faire lire ce qu'on vient de faire dire.

Piga et Bika, CP1, EDICEF NEA, 1984, p. 48.

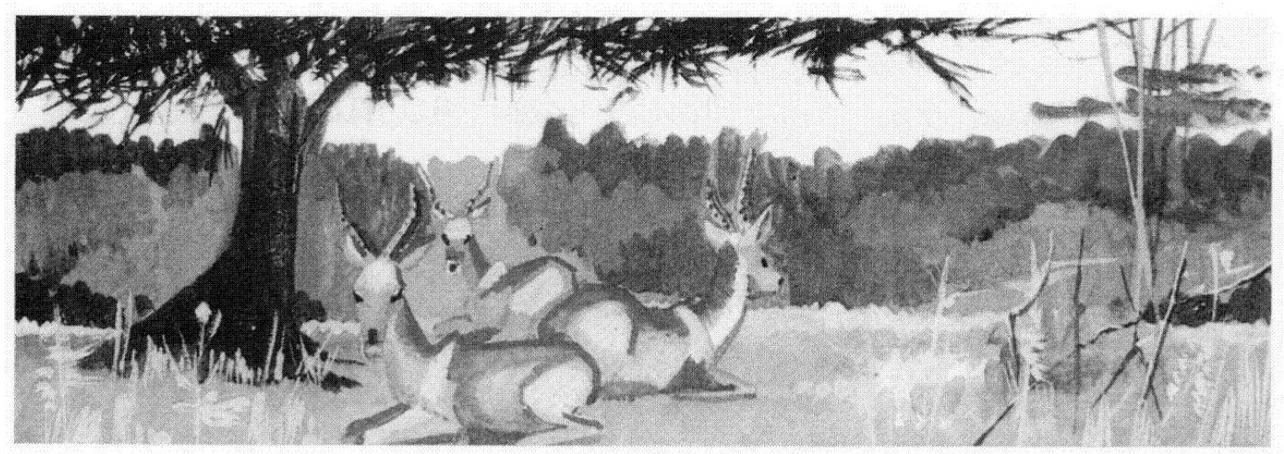

\section{Liaisons méthodologiques}

\subsection{L'écrit transposition de l'oral}

La méthode $A$ toi de parler! élaborée pour Madagascar s'efforce de trouver une autre modalité de liaison. A l'instar des méthodes phonologiques utilisées en France dans la lignée du Sablier, la leçon de lecture s'appuie sur une comptine et c'est cette comptine qui doit jouer le rôle d'articulation entre l'oral et l'écrit : elle permet l'identification des sons et sert de base aux activités de déchiffrage. Toutefois elle est bizarrement sans rapport avec le contenu de la leçon de langage qui s'appuie, elle, sur une sorte de bande dessinée.

\subsection{L'acte de langage-pivot}

Le manuel Le Cabri et le Chacal C.I., élaboré à Djibouti pour la première année d'école primaire, ne se contente pas d'un lien thématique entre le langage et la lecture. La liaison est assurée par l'acte de langage qui fait l'unité de la leçon et permet de conserver en lecture l'orientation communicative qui préside à l'enseignement du langage.

Ainsi, lorsqu'on apprend à se présenter - ce qui est un des premiers objectifs habituels à ce type de méthodes - la lecture concerne le nom du maître à la porte de la classe et la fiche scolaire d'inscription. Assez souvent l'unité de l'acte de langage s'accompagne d'une unité thématique. Par exemple, au dossier 4 , unité 2 , sur le thème "Je voyage ", les conseils de prudence à l'oral se prolongent à l'écrit par la lecture de pancartes du type "Défense de parler au conducteur ». A notre connaissance, Le Cabri et le Chacal C. I. est le premier manuel à avoir ainsi fait de l'acte de langage le pivot de l'articulation langagelecture. Et de ce point de vue l'ouvrage s'oppose aux autres manuels qui ont emprunté à 
l'approche communicative un programme d'objectifs langagiers définis en termes d'actes de parole mais qui renoncent ensuite à considérer que lire est une activité langagière et à en inclure l'apprentissage dans une démarche communicative.

21 Mon livre unique de français adapté au Burundi sous le titre Pour apprendre le français illustre parfaitement cette contradiction entre le point de vue adopté pour le langage et celui de la lecture. Soit l'exemple de la leçon 10 : alors que l'objectif langagier est de «s'informer sur un itinéraire, décrire un itinéraire", le programme en lecture est constitué par : "C'est l'école. Voilà un élève. Voilà les élèves. Voilà un petit élève. » La mise en page traduit parfaitement l'abandon en lecture de l'orientation choisie en langage : on tourne la page pour passer à la lecture, on change de cadre, de personnages, de situation... et de fondements linguistiques.

\subsection{Langage et bande dessinée}

Le souci d'articuler langage-lecture autour de l'acte de langage se retrouve dans le livre djiboutien du C.P. Mais, à ce niveau, c'est la bande dessinée qui est choisie pour effectuer la liaison langage-lecture. B. Maurer ${ }^{1}$ a justifié cette utilisation destinée à assurer les nécessaires imbrications des différentes progressions en langage et en lecture.

La bande dessinée permet, explique-t-il, d'éviter de «cloisonner les différents apprentissages », elle favorise "de constants va-et-vient entre les activités»; elle présente à l'élève " une situation de communication amenant les personnages à utiliser différents actes de langages ». Mais par son caractère écrit elle sert aussi « de support aux activités de lecture ». Ainsi se trouve dépassée la contradiction des méthodes qui donnent à lire de l'oral avec des phrases tirées de dialogues. Document authentique, la bande dessinée réunit habilement dans ses didascalies encadrées et dans les phrases des bulles un écrit et un oral à retrouver et à lire.

Dans le manuel, enfin, le travail de compréhension fait pour la bande dessinée prépare la lecture d'un résumé qui reprend sans les répéter, mais sous une forme véritablement textuelle, des éléments acquis pendant la phase précédente.

Ainsi, par la sélection d'un acte de langage vu sous ses modalités orale et écrite, et grâce à la bande dessinée, langage et lecture sont ici étroitement imbriqués. 
Bande dessinée, langage et lecture.

Le Cabri et le Chacal, CP, EDICEF CRIPEN, Djibouti, 1994, p. 50.

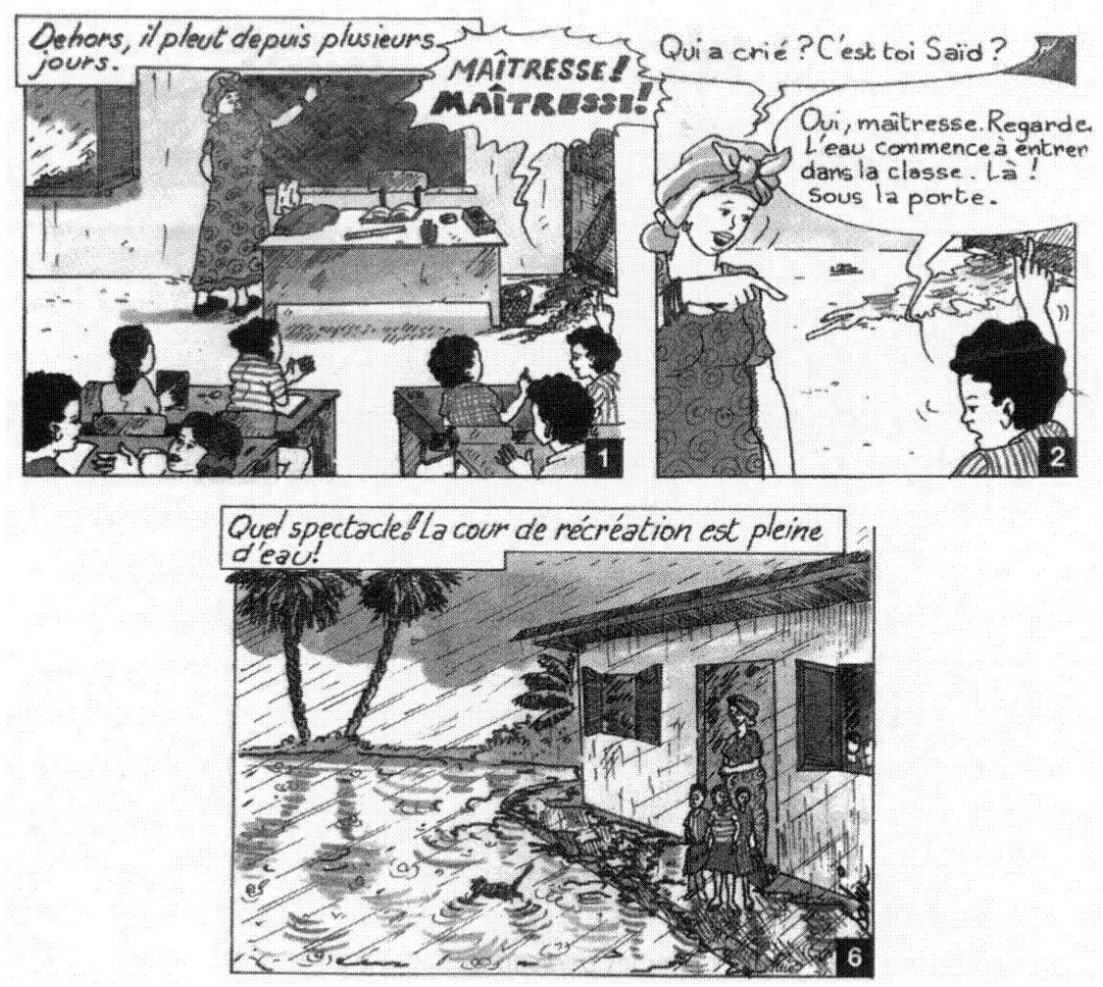

\section{L'écrit dans le langage}

D'autres pistes sont à explorer, dans cette recherche des liaisons entre l'oral et l'écrit, qui ne vont pas forcément dans le sens d'un oral appliqué à l'écrit. N. Marty avait rassemblé en 1991 dans un numéro d'Etudes de linguistique appliquée ${ }^{2}$, des exemples dessinant « des situations interactives, d'entretiens et d'échanges, orientées principalement vers l'amélioration des conduites langagières, à l'écrit ». Mais si les exemples d'interaction entre l'oral et l'écrit abondent dans la vie courante, ils sont par contre plus rares dans les manuels examinés. La méthode Langage en fête CP1 lie en quelque sorte par anticipation les deux enseignements du langage et de la lecture. Alors même que cet ouvrage ne vise aucunement l'enseignement de la lecture et se définit comme un manuel spécifique de langage, il tisse des liens subtils entre les deux apprentissages du parler et du lire.

C'est d'abord l'image, support des échanges oraux, qui assure la présence de l'écrit. La représentation iconique du monde a souvent retenu des éléments porteurs d'écrits. Dans la leçon 1 par exemple, le cadre est celui de l'école, et la découverte de l'image conduit au panneau « Ecole », à la porte du bureau du « Directeur » et aux premiers écrits du tableau noir. Plus loin, un vague terrain de sport est cerné d'une palissade chargée de publicités écrites. Ainsi plus d'une série d'images sur deux met en scène de l'écrit. La pédagogie de la compréhension qui amène à l'interprétation des échanges à partir de questions posées sur l'image permet de faire faire des hypothèses sur ces éléments écrits du cadre et de mettre en place des processus de compréhension langagière à l'oral et à l'écrit. 

L'écrit est parfois au centre de l'action : une petite cuisinière en herbe confond le sel et le sucre et regrette de ne pas savoir lire (thème 4). Ailleurs la clef d'un mystère est expliquée dans un livre par l'adulte qui sait lire (thème 5). Un petit étourdi éprouve tout l'intérêt d'un message écrit pour une communication à distance (thème 5). Ainsi discrètement, sans lourd discours didactique, mais à plusieurs reprises le manuel illustre l'intérêt et la nécessité de savoir lire. Il profite de l'enseignement spécifique du langage pour introduire l'écrit et pour motiver l'apprentissage de la lecture. La méthode de langage fait vivre l'écrit dans quelques-unes de ses fonctions et compense ainsi peut-être en partie la pauvreté scripturale du milieu social et familial de beaucoup d'enfants africains. Et par ses mises en scène de la lecture elle cherche à pallier l'absence de modèle de lecteurs. Ce faisant, l'ouvrage renoue avec une tradition ancienne des manuels du début du siècle qui sélectionnaient dans leurs illustrations des scènes de lecture familiale ou publique ${ }^{3}$.

Enfin dans le droit fil des thèses de certains psychologues cognitivistes (voir la bibliographie de M. Verdelhan-Bourgade en page 46), la méthode installe petit à petit un ensemble de connaissances sur le fonctionnement de la langue à différents niveaux, connaissances métalinguistiques sur lesquelles pourra prendre appui l'enseignement de la lecture. A travers les comptines, les bouts rimés, les jeux d'inventions verbales, les enfants sont familiarisés avec les manipulations des unités phonétiques. Puis des exercices spécifiques, sous les rubriques. "J'apprends les mots» et «Je construis des phrases ", entraînent les élèves à isoler les unités lexicales et les structures. Bref, tout en apprenant àparler, les élèves se dotent des savoirs qui permettront d'inscrire l'apprentissage de la lecture dans le prolongement de ces acquisitions.

Les maîtres ne disposent pas en France d'outils adaptés àla double contrainte d'un enseignement du langage et de la lecture pour des enfants d'origine étrangère. Or la liaison langage-lecture perçue comme une nécessité par les pédagogues s'avère délicate et complexe àétablir. Certes elle doit exister d'abord au plan du contenu thématique, afin $d$ 'ancrer l'apprentissage du lire sur une connaissance du monde. Mais cela ne saurait suffire. Quand un programme d'actes de langage rythme le travail sur l'oral, on ne peut oublier que certains de ces actes de parole ont des réalisations écrites spécifiques dans d'autres situations de communication. De plus, l'accès àl'écrit mobilise des savoirs sur la langue et des processus de compréhension qu'un enseignement adapté du langage peut contribuer à mettre en place. 


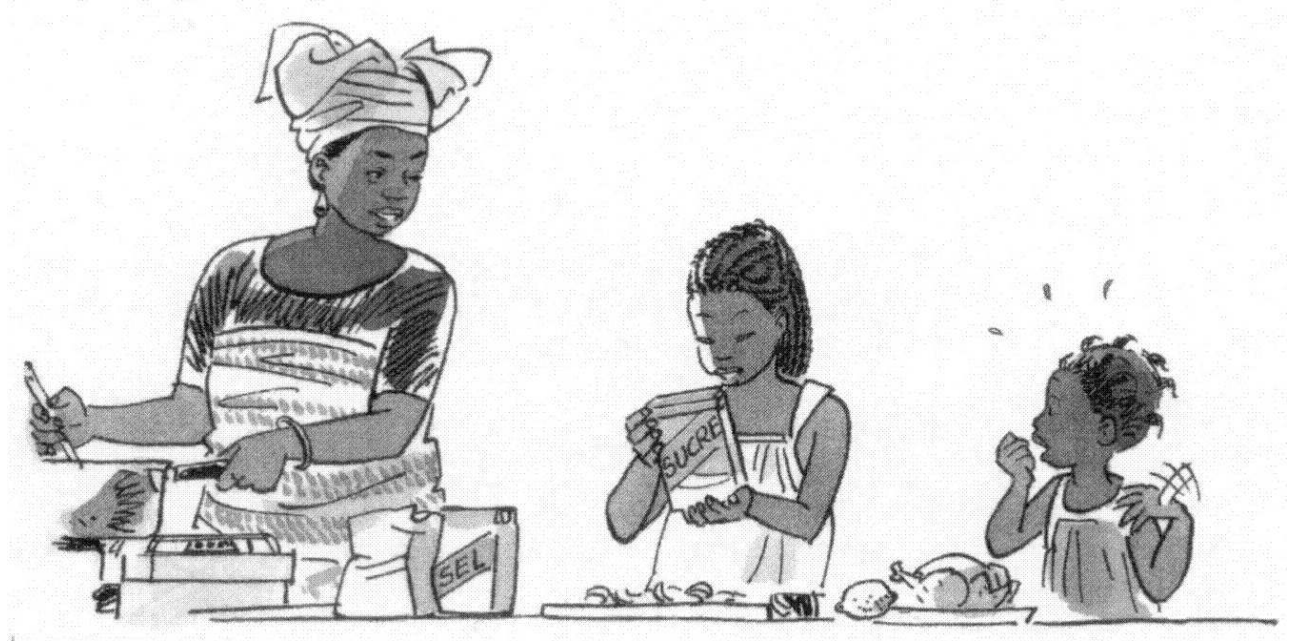

\section{NOTES}

1. MAURER B., «Concevoir un manuel de Français ». in Travaux de didactique du FLE, n 30, 1993.

2. N. MARTY coord., "L'écrit dans l'oral ", Études de linguistique Appliquée, n 81, Paris. DidierErudition, 1991.

3. CHARTIER A.-M \& HEBRARD J., Discours sur la lecture. Paris, Centre Georges Pompidou, 1989.

\section{RÉSUMÉS}

Les manuels destinés aux cours préparatoires des pays de l'Afrique francophone ont à résoudre le problème de la liaison langage - lecture. Leur examen montre que cette liaison est réalisée selon différentes modalités plus ou moins superficielles. Si certains se contentent d'une juxtaposition ou d'une répétition en utilisant souvent la légende de l'image, d'autres établissent de subtiles liaisons méthodologiques autour de l'acte de langage et par la mise en place de connaissances linguistiques.

The schoolbooks intended for use in the primary grades in french speaking countries of Africa must resolve the problem of the link between language and reading. An examination of these books shows that this link is accomplished by various means, these being more of less superficial. Though some publishers content themselves with juxtaposition or repetition oft-times using the captions of pictures, others establish subtle methodological links around the act of speaking and through the acquisition of linguistic knowledge. 
INDEX

Mots-clés : Afrique francophone, cours préparatoire, langage, lecture, manuel scolaire

Keywords : french speaking Africa, language, primary level, reading, schoolbook

\section{AUTEUR}

MICHEL VERDELHAN

Professeur, IUFM de Montpellier 